\title{
The Contribution of Common Surgically Implanted Hardware to Functional MR Imaging Artifacts
}

\author{
A.A. Desai, M.K. Strother, C.C. Faraco, V.L. Morgan, T.R. Ladner, L.M. Dethrage, L.C. Jordan, and M.J. Donahue
}

\begin{abstract}
BACKGROUND AND PURPOSE: Blood oxygenation level-dependent MR imaging is increasingly used clinically to noninvasively assess cerebrovascular reactivity and/or language and motor function. However, many patients have metallic implants, which will induce susceptibility artifacts, rendering the functional information uninformative. Here, we calculate and interpret blood oxygenation leveldependent MR imaging artifact impact arising from surgically implanted hardware.
\end{abstract}

MATERIALS AND METHODS: A retrospective analysis of all blood oxygenation level-dependent MRIs $(n=343 ; \mathrm{B} 0=3 \mathrm{~T} ; \mathrm{TE}=35 \mathrm{~ms}$; gradient echo EPI) acquired clinically (year range $=2006-2014$ ) at our hospital was performed. Blood oxygenation level-dependent MRIs were most commonly prescribed for patients with cerebrovascular disease $(n=80)$ or patients undergoing language or motor localization $(n=263)$. Artifact volume (cubic centimeters) and its impact on clinical interpretation were determined by a board-certified neuroradiologist.

RESULTS: Mean artifact volume associated with intracranial hardware was $4.3 \pm 3.2 \mathrm{~cm}^{3}\left(\right.$ range $\left.=1.1-9.4 \mathrm{~cm}^{3}\right)$. The mean artifact volume from extracranial hardware in patients with cerebrovascular disease was $28.4 \pm 14.0 \mathrm{~cm}^{3}$ (range $\left.=6.1-61.7 \mathrm{~cm}^{3}\right)$, and in patients with noncerebrovascular disease undergoing visual or motor functional mapping, it was $39.9^{3} \pm 27.0 \mathrm{~cm}^{3}\left(\right.$ range $\left.=6.9-77.1 \mathrm{~cm}^{3}\right)$. The mean artifact volume for ventriculoperitoneal shunts was $95.7 \pm 39.3 \mathrm{~cm}^{3}\left(\right.$ range $\left.=64.0-139.6 \mathrm{~cm}^{3}\right)$. Artifacts had no-to-mild effects on clinical interpretability in all patients with intracranial implants. Extracranial hardware artifacts had no-to-moderate impact on clinical interpretability, with the exception of 1 patient with 12 KLS-Martin maxDrive screws with severe artifacts precluding clinical interpretation. All examined ventriculoperitoneal shunts resulted in moderate-to-severe artifacts, limiting clinical interpretation.

CONCLUSIONS: Blood oxygenation level-dependent MR imaging yields interpretable functional maps in most patients beyond a small $\left(30-40 \mathrm{~cm}^{3}\right)$ artifact surrounding the hardware. Exceptions were ventriculoperitoneal shunts, particularly those with programmable valves and siphon gauges, and large numbers of KLS-Martin maxDrive screws.

ABBREVIATIONS: $B$ OLD $=$ blood oxygenation level-dependent; $C V R=$ cerebrovascular reactivity; $\mathrm{VP}=$ ventriculoperitoneal

B lood oxygenation level-dependent (BOLD) fMRI is becoming increasing used for presurgical mapping of eloquent cortex before resection of brain tumors ${ }^{1}$ or epileptogenic foci ${ }^{2}$ and to assess cerebrovascular reactivity (CVR) when combined with hypercapnic gas stimuli. ${ }^{3,4}$ The main advantage of BOLD fMRI is the lack of ionizing radiation or exogenous contrast required, which makes it a par-

Received October 22, 2014; accepted after revision March 26, 2015.

From the Departments of Radiology and Radiological Sciences (A.A.D., M.K.S., C.C.F., V.L.M., T.R.L., L.M.D., M.J.D.), Pediatrics (L.C.J.), Division of Pediatric Neurology, Psychiatry (M.J.D.), and Neurology (M.J.D.), Vanderbilt University School of Medicine, Nashville, Tennessee; and Department of Physics and Astronomy (M.J.D.), Vanderbilt University, Nashville, Tennessee.

This work was supported by the National Institutes of Health/National Institute of Neurological Disorders and Stroke (5R01NS078828) and the American Heart Association (14GRNT20150004).

Paper previously presented at: American Society of Neuroradiology Annual Meeting and the Foundation of the ASNR Symposium, May 17-22, 2014; Montreal, Quebec, Canada. ticularly appealing approach for longitudinal monitoring of patients or for evaluating short-term responses to therapy in situations in which exogenous contrast agents may be dose-restricted.

A known limitation of BOLD fMRI over alternative approaches for assessing hemodynamics is the sensitivity of BOLD to magnetic field inhomogeneities, including those produced by implants. At clinical field strengths of $1.5 \mathrm{~T}$ to $3 \mathrm{~T}$, BOLD fMRI requires a relatively long $\mathrm{TE}(30-55 \mathrm{~ms})$ to sensitize the sequence to susceptibility differences between oxygenated (diamagnetic) and deoxygenated (paramagnetic) blood. However, this require-

Please address correspondence to Aditi A. Desai, MD, Department of Radiology and Radiological Sciences, 1161 21st Ave South, Medical Center North, Suite CCC1121, Nashville, TN 37232-2675; e-mail: aditi.desai@vanderbilt.edu

- Indicates open access to non-subscribers at www.ajnr.org

三 Indicates article with supplemental on-line tables.

http://dx.doi.org/10.3174/ajnr.A4419 
ment also sensitizes the sequence to susceptibility variations between implants and surrounding tissue. This feature adds variability to the static magnetic field (B0) and may manifest as distortion or signal voids.

The influence of implants on the quality of the diagnostic information has been historically defined as "MR imaging compatibility." Medical devices labeled "MR imaging compatible" were MR imaging-safe and did not affect the quality of the diagnostic information. However, due to ongoing confusion with medical labeling, in 2005, the US Food and Drug Administration recognized a new set of terms by the American Society for Testing and Materials international, which included the influence of implants on image quality, and requested manufacturers to use the terminology for new products. However, there remains a fundamental need to define the extent of image artifacts, because manufacturers frequently provide only general statements regarding the possible impact on image quality. ${ }^{5}$ Furthermore, such MR imaging compatibility is most commonly considered in the context of conventional structural MR imaging sequences that use short TEs, ${ }^{6-8}$ avoid single-shot echo-planar imaging readouts, and/or may not use parallel imaging, all of which are commonly used in BOLD fMRI. Thus, the impact of implants specifically on BOLD fMRI signal quality is not well-characterized.

For cerebral hemodynamic measurements derived from BOLD MR imaging to be reliable in routine clinical practice, susceptibility-induced influences of endovascular and surgical hardware on BOLD MR imaging must be characterized, particularly in studies that require imaging before and after implantation of surgical hardware. The purpose of this study was to review BOLD fMRI examinations performed clinically at $3 \mathrm{~T}$ and to calculate the volume and artifact extent in patients with implants, as well as the degree to which the artifact impacts the clinical utility of the study. This information is intended to serve as an exemplar for when BOLD fMRI scans may remain interpretable, even in the presence of surgically implanted hardware.

\section{MATERIALS AND METHODS \\ Patient Demographics and Ethical Considerations}

This study was approved by our local institutional review board and conformed to the requirements of the United States Health Insurance Portability and Accountability Act. We conducted a retrospective analysis of all BOLD MRI acquired clinically (January 2006 to March 2014) at our institution as part of 2 separate institutional review board-approved studies. In one study, patients were prospectively recruited to undergo BOLD MR imaging by using a CVR protocol (institutional review board No. 110468, $n=80$ ). Additionally, clinical BOLD MR imaging was performed by using a protocol with functional tasks for language or motor localization (functional localizer protocol, $n=263$ ). An institutional review board exemption was obtained to retrospectively review data from the functional localizer protocol (institutional review board No. 150190).

Implant manufacturer and model were obtained when available in the electronic medical record, and all patients were screened per departmental policy to determine the MR imaging compatibility and safety of implants before scanning, following the American College of Radiology recommendations for safe MR imaging practices. ${ }^{9}$

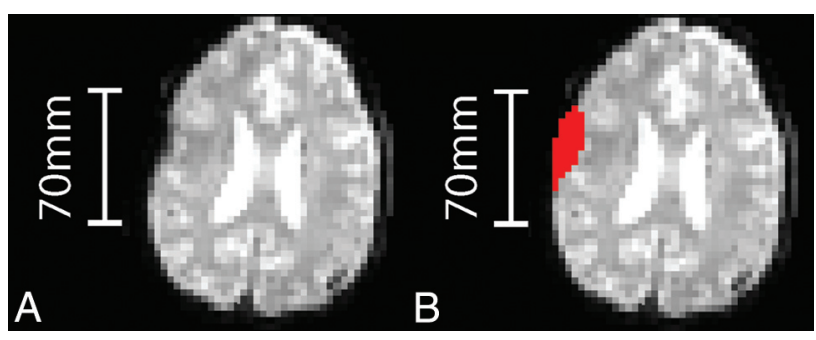

FIG 1. Representative images of artifact masks, which colocalize with areas of signal loss. A, A representative section from the magnitude gradient echo BOLD fMRI volume in a patient with right-sided surgical closure hardware. $B$, The artifact mask (red). The volume of the mask was summed over all sections to quantify the total artifact volume.

\section{MR Imaging Parameters}

All patients in the CVR protocol were scanned by using 3T MR imaging (Achieva; Phillips Healthcare, Best, the Netherlands) with body coil transmission and sensitivity encoding 8 -array coil reception with a multimodal protocol, which included hypercarbic BOLD: spatial resolution $=3.4 \times 3.4 \times 5 \mathrm{~mm}^{3}$, single-shot gradient-echo EPI, $\mathrm{TR} / \mathrm{TE}=2000 / 35 \mathrm{~ms}$, flip angle $=80^{\circ}$, sensitivity encoding factor $=$ 2, 30 sections; and a block paradigm of $3 / 3$ minutes baseline (room air) $/ 5 \%$ carbogen $\left(5 \% \mathrm{CO}_{2}, 95 \% \mathrm{O}_{2}\right)$ breathing repeated twice. Patients undergoing the functional localizer protocol were scanned by using the same scanner with a slightly modified protocol: spatial resolution $=3.75 \times 3.75 \times 5.00 \mathrm{~mm}^{3}$, single-shot gradient-echo EPI, $\mathrm{TR} / \mathrm{TE}=2000 / 35 \mathrm{~ms}$, flip angle $=78^{\circ}$, sensitivity encoding factor $=$ $1.8,30$ sections. The TE and readout type (single-shot gradient-echo EPI) were identical for both protocols; the total voxel volume (57.8 versus $52.7 \mathrm{~mm}^{3}$ ) and parallel imaging factor (2.0 versus 1.8$)$ were nearly identical between protocols and within the range of common fMRI acquisition parameters (see "Discussion").

\section{Analysis}

All fMRI data were corrected for motion and baseline drift and were coregistered to T1 and standard space (Montreal Neurological Institute, $2 \mathrm{~mm}$ ) by using linear registration routines from the fMRI of the Brain Software Library (FSL; http://www.fmrib.ox. ac.uk/fsl). ${ }^{10}$ Dephasing artifacts associated with metallic objects on BOLD MR imaging were overseen by a board-certified neuroradiologist (M.K.S. with 13 years' experience) and were quantified on all patients with implanted hardware by manually drawing ROIs demarcating areas of signal loss on each affected image section (Fig 1). These data were then summed to yield a total artifact volume (cubic centimeters). In patients with multiple ipsilateral implants, artifact volume per hemisphere was summated, because implant proximity precluded the ability to distinguish the extent of artifacts associated with each individual implant in subjects with bilateral implants. In patients with bilateral implants, right and left hemisphere artifacts were calculated separately. Additional analysis was performed to calculate the time course signal-to-noise ratio in each voxel by measuring the ratio of the mean baseline signal to the $\mathrm{SD}$ of the baseline signal with time.

All BOLD MRIs were reviewed by a fellowship-trained, boardcertified neuroradiologist (M.K.S) and a MR imaging physicist (V.L.M. with 15 years' experience) to characterize the degree that artifact volume impacted clinical interpretability by using the following scale: none $=$ no compromise of functional interpretation AJNR Am J Neuroradiol 36:2068-73 Nov 2015 www.ajnr.org 


\begin{tabular}{|c|c|c|c|c|}
\hline Patient, Location, and Implant Type & Model & Material & $\begin{array}{l}\text { Total Volume }\left(\mathrm{cm}^{3}\right) \\
\text { from Artifacts }\end{array}$ & $\begin{array}{l}\text { Impact on Clinical } \\
\text { Interpretation }\end{array}$ \\
\hline Patient 1 & & & 3.1 & Mild \\
\hline MCA stent & Wingspan stent ${ }^{\mathrm{a}}$ & Titanium, nickel alloy & & \\
\hline Patient 2A & & & 9.4 & None \\
\hline ICA stent & Unknown & Unknown & & \\
\hline Anterior choroidal artery coils & Unknown & Unknown & & \\
\hline Patient 2B & & & 8.7 & None \\
\hline ICA bifurcation aneurysm clip & Yasargil clip ${ }^{\mathrm{b}}$ & Titanium & & \\
\hline Patient 3 & & & 2.1 & Mild \\
\hline MCA stent & Pharos Vitesse stent & Titanium, nickel alloy & & \\
\hline Patient 4 & & & 4.7 & Mild \\
\hline \multirow{4}{*}{$\begin{array}{l}\text { Posterior communicating artery } \\
\text { aneurysm coils }\end{array}$} & Matrix Soft coils ${ }^{c}$ & Platinum & & \\
\hline & Matrix Ultrasoft coils ${ }^{c}$ & Platinum & & \\
\hline & $\begin{array}{l}\text { Guglielmi detachable coil-10 } \\
\text { Soft coils }{ }^{c}\end{array}$ & Platinum & & \\
\hline & Sapphire Tension Safe coils ${ }^{d}$ & Platinum & & \\
\hline ICA stent & Neuroform EZ stent ${ }^{c}$ & Titanium, nickel alloy & & \\
\hline Patient 5 & & & 1.1 & None \\
\hline $\begin{array}{l}\text { Anterior communicating artery } \\
\text { aneurysm coils }\end{array}$ & Target 360 coils $^{c}$ & Platinum & & \\
\hline
\end{tabular}

\footnotetext{
${ }^{a}$ Manufactured by Stryker, Kalamazoo, Michigan for Boston Scientific, Natick, Massachusetts

${ }^{\mathrm{b}}$ Aesculap, Center Valley, Pennsylvania.

' Stryker, Kalamazoo, Michigan.

${ }^{\mathrm{d}}$ Micro Therapeutics, Irvine, California.
}

Table 2: Artifacts from surgically implanted hardware

\begin{tabular}{lccc}
\multicolumn{1}{c}{ Hardware Type } & $\begin{array}{c}\text { Average Artifact } \\
\text { Volume }\left(\mathbf{c m}^{\mathbf{3}}\right)\end{array}$ & SD & Range $\left(\mathbf{c m}^{\mathbf{3}}\right)$ \\
\hline Intracranial hardware & 4.3 & 3.2 & $1.1-9.4$ \\
Extracranial hardware (CVR protocol) & 28.4 & 14.0 & $6.1-61.7$ \\
Extracranial hardware (functional localizer protocol) & 39.9 & 27.0 & $6.9-77.1$ \\
Ventriculoperitoneal shunt & 95.7 & 39.3 & $64.0-139.6$ \\
\hline
\end{tabular}

from hardware artifact (eg, hardware artifacts do not project onto the parenchyma); mild = hardware artifacts present but do not compromise clinical interpretation; moderate $=$ hardware artifacts somewhat limit, but do not preclude, clinical interpretation; severe $=$ hardware artifacts preclude clinical interpretation.

\section{RESULTS}

In patients in the CVR protocol, 26 of 80 (32.5\%) had surgical hardware: intracranial implants $(n=5)$, extracranial hardware $(n=19)$, and ventriculoperitoneal (VP) shunts $(n=2)$. Intracranial implant characteristics are summarized in Table 1. Implanted extracranial hardware (On-line Table 1) primarily included closure hardware composed of titanium alloy and 1 patient with a polyethylene implant. Six of 19 patients had bilateral closure hardware with artifact sizes calculated separately by hemisphere, for a total of 25 hemispheres. Implanted VP shunts are described in On-line Table 2. Artifacts from surgically implanted hardware are shown in Table 2.

In patients in the functional localizer protocol, 14 of 263 (5.3\%) had surgical hardware: extracranial hardware $(n=13)$ and VP shunt $(n=1)$. Extracranial hardware comprised surgical closure hardware, and implant records were not available for these patients. The implanted VP shunt is included in On-line Table 2 .

Mean artifact volume associated with intracranial hardware in patients undergoing the CVR protocol was $4.3 \pm 3.2 \mathrm{~cm}^{3}$; range $=$ $1.1-9.4 \mathrm{~cm}^{3}$. As a point of reference, the average volume of the adult human brain is approximately $1380 \mathrm{~cm}^{3} .{ }^{11}$ The small artifact volume associated with implanted intracranial hardware did not compromise the functional interpretation of cerebral hemodynamic data, with intracranial implants considered to have no $(n=3)$ or mild $(n=3)$ effect on the clinical interpretability of the examination in the affected hemisphere. Figure 2 shows a representative image maximally affected by implanted intracranial hardware and hemodynamic data from a patient with a left MCA stent with in-stent restenosis, demonstrating the impact of the artifacts on the reactivity map.

Mean artifact volume of extracranial hardware in patients in the CVR protocol was $28.4 \pm 14.0 \mathrm{~cm}^{3}$; range $=6.1-61.7 \mathrm{~cm}^{3}$; and in patients in the functional localizer protocol, it was $39.9 \pm$ $27.0 \mathrm{~cm}^{3}$ (range $\left.=6.9-77.1 \mathrm{~cm}^{3}\right)$. Artifact volume associated with titanium closure hardware is typically small and peripheral, with 19 of the evaluated hemispheres (76\%) considered to have mild artifacts, which did not compromise clinical interpretation of cerebral hemodynamics (Fig 3). Closure hardware artifacts in 5 of the evaluated hemispheres $(20 \%)$ had a moderate effect on the clinical interpretation. However, in the patient (patient 10) with the largest number of maxDrive screws (KLS-Martin, Jacksonville, Florida), ${ }^{12}$ artifact size $\left(61.7 \mathrm{~cm}^{3}\right)$ was rated as severe and precluded ipsilateral CVR interpretation in the region of the indirect revascularization that underlay the closure hardware (Fig 4). These artifacts were larger than those in other patients with fewer KLS-Martin LP maxDrive screws (patient 20: 5 screws, artifact volume $=17.1 \mathrm{~cm}^{3}$; 


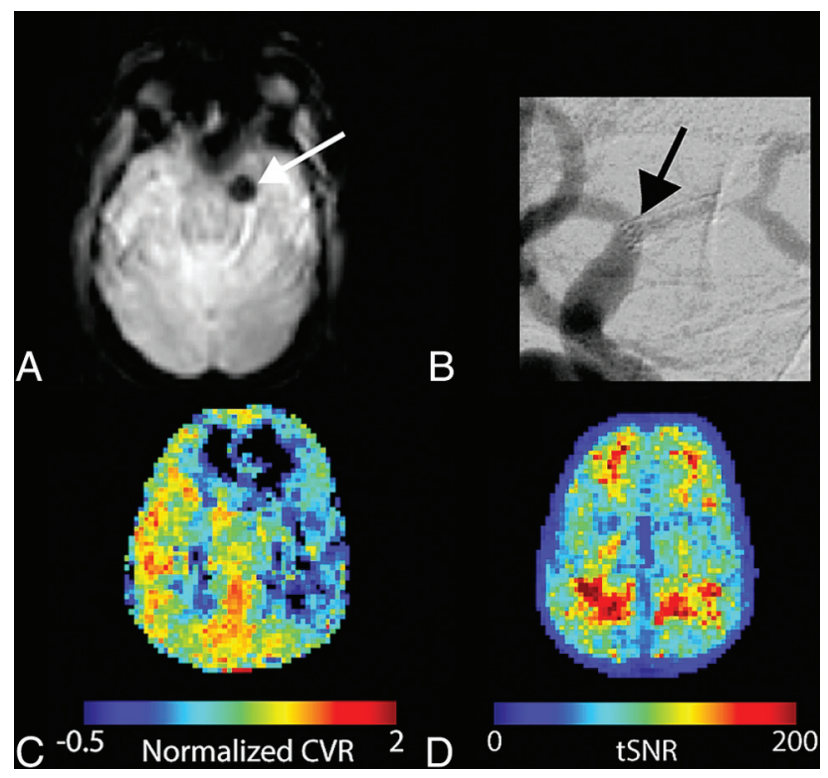

FIG 2. A representative patient (patient 3) with an intracranial implant. Signal drop-out from a left MCA Pharos Vitesse stent (Codman Neurovascular) is apparent on the magnitude BOLD fMRI image (A, white arrow), resulting in a total artifact volume of $2.1 \mathrm{~cm}^{3}$, which only mildly affected clinical interpretation of the examination. The patient was evaluated 2 years following implantation of the Pharos Vitesse stent in a stenosed left MCA. DSA (B) shows in-stent restenosis (black arrow), with corresponding decreased cerebrovascular reactivity (normalized CVR: voxel CVR normalized to cerebellar CVR) in the left MCA territory $(C)$. In contrast, there is relative symmetry of the temporal signal-to-noise ratio (tSNR) map (D), suggesting that the asymmetric hemodynamic findings are not attributable to artifacts.

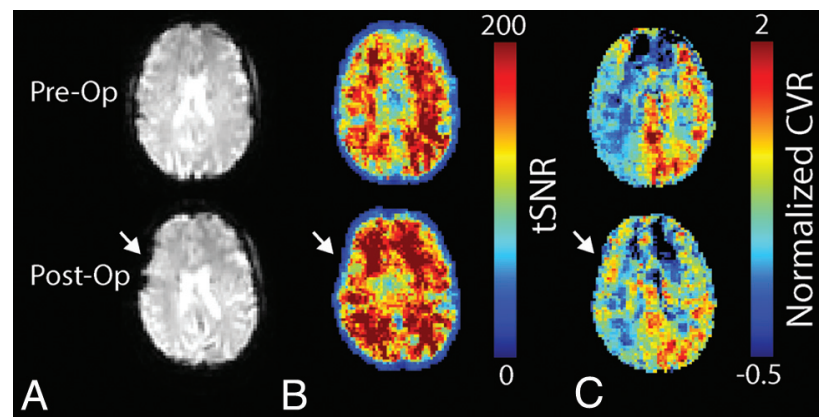

FIG 3. A patient with Moyamoya disease and asymmetric right-sided intracranial stenosis who underwent right encephaloduroanrteriosynangiosis. Comparison of pre- (Pre-Op) and postoperative (PostOp) magnitude BOLD images $(A)$ shows minimal artifacts $\left(26.5 \mathrm{~cm}^{3}\right)$ associated with the closure hardware (LP Plate $0.6 \times 15 \mathrm{~mm}$, Low Profile Micro Plate, and Cross-Drive $1.5 \times 4 \mathrm{~mm}$ screws; KLS-Martin) at the corresponding surgical site, which was determined to only mildly impact clinical interpretation of the study. Both pre- and postoperative temporal signal-to-noise ratio (tSNR) maps (B) demonstrate relatively symmetric tSNR, while pre- and postoperative CVR maps $(C)$ show decreased CVR in the right hemisphere along the right frontal cortex, which improves following revascularization.

patient 21: 8 screws, artifact volume $=24.6 \mathrm{~cm}^{3}$ ). The number of screws did not correlate directly with artifact volume across screw types, with up to 19 LP Cross-Drive screws (KLS-Martin) in 1 patient causing fewer artifacts $\left(23.5 \mathrm{~cm}^{3}\right)$ than the 12 KLS-Martin maxDrive screws $\left(61.7 \mathrm{~cm}^{3}\right)$.

The average artifacts associated with VP shunts were $95.7 \pm$ $39.3 \mathrm{~cm}^{3}$, range $=64.0-139.6 \mathrm{~cm}^{3}$, and were rated as severe in 2 of

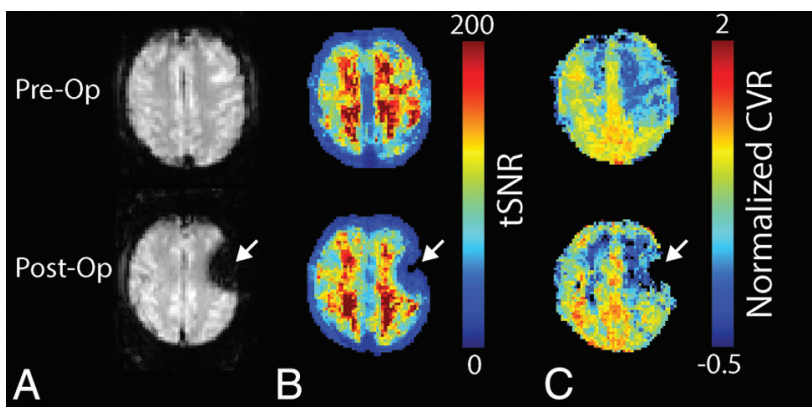

FIG 4. Pre- (Pre-Op) and postoperative (Post-Op) magnitude and hemodynamic images in a patient with left-sided idiopathic Moyamoya disease who underwent a left encephaloduroarteriosynangiosis with implantation of closure hardware. Magnitude images $(A)$ demonstrate the large artifacts $\left(61.7 \mathrm{~cm}^{3}\right)$ associated with surgical closure hardware, attributable to the large number of KLS-Martin maxDrive screws. Artifacts were thought to severely impact clinical interpretation of the study, and hemodynamic evaluation with temporal signal-to-noise ratio maps $(B)$ and reactivity maps $(C)$ shows that interpretable hemodynamic data were not obtainable near the closure hardware. TSNR indicates temporal signal-to-noise ratio.

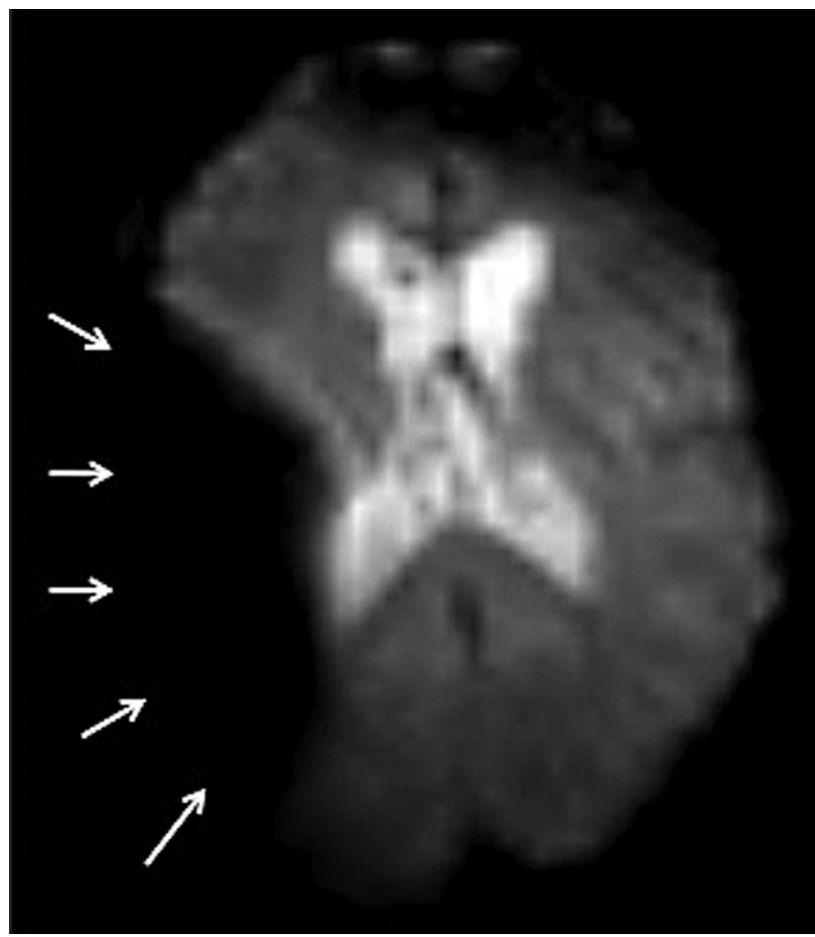

FIG 5. Artifacts (white arrows, volume $=139.6 \mathrm{~cm}^{3}$ ) associated with a ventriculoperitoneal shunt with a Certas programmable valve and siphon gauge (Codman and Shurtleff).

3 patients (Fig 5, CVR protocol) and moderate in the remaining patient (functional localizer protocol).

\section{DISCUSSION}

\section{Specificity of Different Implants on BOLD fMRI Artifact Volume}

Dephasing artifacts on MR imaging associated with implanted devices are caused by perturbations in the magnetic field due to differences in magnetic susceptibility or the degree of magnetization of an object in the presence of a magnetic field between the implanted device and surrounding tissue. Larger differences in 
magnetic susceptibility between an implanted device and surrounding tissue lead to larger perturbations of the magnetic field and greater spectral dispersion of spins within a voxel, resulting in more extensive dephasing artifacts. In BOLD fMRI, long-TE, T2* weighted imaging is used to sensitize the sequence to susceptibility differences between oxygenated and deoxygenated blood, but these imaging parameters also render the sequence exquisitely sensitive to susceptibility variations from magnetic field inhomogeneities, exacerbating artifact extent. ${ }^{6-8}$ The effect of increased dephasing artifacts associated with implants on the quality of data obtained specifically from BOLD MR imaging has not been previously well-documented, to our knowledge. Our results demonstrate that BOLD MR imaging is interpretable in the presence of most implants evaluated, except for VP shunts, particularly those with programmable valves and siphon gauges and 1 patient with multiple KLS-Martin maxDrive screws.

The intracranial implants and extracranial hardware analyzed are composed of materials with low magnetic susceptibilities, such as titanium, nickel, platinum, or their alloys. All intracranial implanted devices produced small artifacts that had no-to-mild effects on clinical interpretability, and closure hardware typically resulted in small, peripheral artifacts that did not preclude interpretation. The single notable exception was a patient with a large number of KLS-Martin maxDrive screws, which were associated with more artifacts than the KLS-Martin Cross-Drive screws. While both the maxDrive and Cross-Drive screws are made of a titanium and nickel alloy, the maxDrive screw has a larger head profile. The number of screws did not correlate directly with artifacts across screw types.

In contrast, VP shunts resulted in large artifacts that produced a moderate-to-severe impact on clinical interpretation, an effect attributable to the shunt valve composition. The valve portion of the VP shunt is composed of $316 \mathrm{~L}$ stainless steel in addition to unalloyed titanium and tantalum in some models. ${ }^{12,13}$ Stainless steel contains iron, a ferromagnetic substance. While 316L stainless steel contains a larger amount of nickel, which stabilizes iron in its nonmagnetic state and reduces its magnetic susceptibility, its magnetic susceptibility remains larger relative to titanium, resulting in larger perturbations of the magnetic field and more extensive artifacts. ${ }^{14,15}$ In addition, the Certas programmable valve (Codman \& Shurtleff, Raynham, Massachusetts) contains neodymium magnets, which caused extensive signal loss ${ }^{13,16,17}$ and likely contributed to the large artifact volume $\left(139.6 \mathrm{~cm}^{3}\right)$ relative to other VP shunts evaluated $\left(64.0\right.$ and $\left.83.5 \mathrm{~cm}^{3}\right)$.

Studies have described the effect of previously implanted surgical hardware on BOLD fMRI in the preoperative evaluation of brain tumor resection, demonstrating that language lateralization and primary motor cortex activation can be ascertained despite a reduction in total volume activation due to susceptibility artifacts. ${ }^{18,19}$ Our study confirms the feasibility of obtaining data despite implants, which is particularly important in patients with cerebrovascular disease because longitudinal monitoring of patients before and after revascularization may be desired.

\section{Imaging Parameters and Artifact Volume}

Artifact volume will vary with imaging readout and scan parameters. ${ }^{20}$ We have used scan parameters that are common for
BOLD fMRI on all major vendor platforms, including single-shot gradient-echo EPI, voxel volume $=50-60 \mathrm{~mm}^{3}$, and TE centered on the approximate $3 \mathrm{~T}$ tissue $\mathrm{T} 2{ }^{*}$ (eg, $35 \mathrm{~ms}$ ). ${ }^{21,22} \mathrm{We}$ also used image-based parallel imaging (sensitivity encoding) with an acceleration factor of 1.8-2.0, which reduces the EPI readout train by an approximate factor of 2. Geometric distortions from offresonant spins and signal drop-out are largely affected by susceptibility gradients, and parallel imaging can reduce these issues by increasing the per-voxel bandwidth in the phase-encoding direction, ${ }^{23}$ thereby resulting in a shorter EPI readout (eg, approximately $36 \mathrm{~ms}$ without parallel imaging versus $18 \mathrm{~ms}$ with parallel imaging by using scan parameters in our sequences). While we used sensitivity encoding in our protocol, which is common on Philips scanners, $k$-space parallel imaging by using Generalized Autocalibrating Partial Parallel Acquisition or Autocalibrating Reconstruction for Cartesian sampling is more common on Siemens and GE Healthcare scanners, respectively, and performs comparably for reducing geometric distortions when similar acceleration factors are used. In the absence of parallel imaging, partial $k$-space acquisitions may also be used to reduce geometric distortions.

\section{Limitations}

First, information regarding implant manufacturer and type is not known for all implants considered, precluding a rigorous analysis in all patients. However, the composition of implants evaluated is similar to that in other commonly deployed implants; thus, results may generalize. Of the patients with intracranial implants analyzed, 1 (patient 2A) had incomplete information available regarding embolization coils and an ipsilateral stent, though this patient had an artifact size $\left(9.4 \mathrm{~cm}^{3}\right)$ similar to that in another patient (patient 4A) with known platinum embolization coils and an ipsilateral titanium and nickel alloy stent $\left(4.7 \mathrm{~cm}^{3}\right)$. Indeed, most embolization coils are composed of platinum, with only the earliest models containing stainless steel, ${ }^{24,25}$ and most intracranial stents are composed of titanium, titanium alloys, or stainless steel. ${ }^{24,26,27}$ Thus, our finding that the small artifacts produced by intracranial implants do not impact clinical interpretability of BOLD fMRI is likely generalizable, with the exception of those models of intracranial implants that contain stainless steel, which were not evaluated in our study population and may produce larger artifacts due to higher magnetic susceptibility.

Information regarding specific implant manufacturers and composition was not available for a subset of patients with extracranial implants (CVR protocol, $n=2$; functional localizer protocol, $n=13$ ). However, those patients with unknown closure hardware had artifact extent and clinical interpretability similar to those in patients with known extracranial hardware, which primarily included closure hardware composed of titanium alloy. Most implanted neurosurgical hardware is composed of titanium or titanium alloys similar to those evaluated in our study, ${ }^{24,25}$ suggesting that results are likely generalizable. We did not evaluate any issues related to the safety of metallic devices; however, this topic has been studied extensively, and all patients were screened by using standard MR imaging safety screening procedures. ${ }^{9}$

Second, while BOLD fMRI is the most common noninvasive 
fMRI application, additional methods such as arterial spin-labeling are being increasingly used. ${ }^{28}$ We did not specifically evaluate the impact of artifacts on arterial spin-labeling image quality; however, arterial spin-labeling generally uses readouts similar to those in BOLD (eg, single-shot EPI with comparable spatial resolution), however with a shorter TE (eg, 10-20 ms). Thus, the impact of image distortion on similar data should be reduced relative to BOLD, and findings from this study could provide a conservative reference for guiding similar studies.

Third, this study focused on patients undergoing BOLD fMRI for clinical purposes, primarily those with cerebrovascular disease, epilepsy, and brain tumors. Thus, implants considered were specific to these populations, and a different population may have a higher fraction of implants not considered in this study. Therefore, while we hope that the findings of this study are useful for guiding imaging decisions in some of the most common types of patients undergoing BOLD fMRI, future studies incorporating a broader range of patients and implant types would be useful.

\section{CONCLUSIONS}

Three-Tesla single-shot gradient-echo EPI BOLD MR imaging performed on patients with a variety of implanted intracranial and extracranial hardware yields interpretable image quality in most patients beyond a small $\left(30-40 \mathrm{~cm}^{3}\right)$ volume surrounding the hardware. Exceptions were VP shunts, particularly those with programmable valves and siphon gauges, and large numbers of KLS-Martin maxDrive screws.

Disclosures: Aditi A. Desai—RELATED: Grant: National Institutes of Health, ${ }^{*}$ Comments: The study was funded by a National Institutes of Health grant. Megan K. Strother-RELATED: Grant: National Institutes of Health/National Institute of Neurological Disorders and Stroke R01 NS078828-01A1.* Carlos C. Faraco-RELATED: Grant: National Institutes of Health, ${ }^{\star}$ Comments: T-32 Postdoctoral Fellow Training Grant. Lori C. Jordan—UNRELATED: Grants/Grants Pending: American Heart Association Collaborative Science Award, ${ }^{*}$ Comments: to study novel MRI techniques in sickle cell disease. Manus J. Donahue-RELATED: Grant: National Institutes of Health grants (National Institutes of Health/National Institute of Neurological Disorders and Stroke and National Institutes of Health/National Institute of Nursing Research).* *Money paid to the institution.

\section{REFERENCES}

1. Håberg A, Kvistad KA, Unsgård G, et al. Preoperative blood oxygen level-dependent functional magnetic resonance imaging in patients with primary brain tumors: clinical application and outcome. Neurosurgery 2004;54:902-14; discussion 914-15

2. Binder JR. Functional MRI is a valid noninvasive alternative to Wada testing. Epilepsy Behav 2011;20:214-22

3. Mandell DM, Han JS, Poublanc J, et al. Mapping cerebrovascular reactivity using blood oxygen level-dependent MRI in patients with arterial steno-occlusive disease: comparison with arterial spin labeling MRI. Stroke 2008;39:2021-28

4. Donahue MJ, Dethrage LM, Faraco CC, et al. Routine clinical evaluation of cerebrovascular reserve capacity using carbogen in patients with intracranial stenosis. Stroke 2014;45:2335-41

5. Shellock FG, Woods TO, Crues JV 3rd. MR labeling information for implants and devices: explanation of terminology. Radiology 2009; 253:26-30

6. Schenck JF. The role of magnetic susceptibility in magnetic resonance imaging: MRI magnetic compatibility of the first and second kinds. Med Phys 1996;23:815-50

7. Port JD, Pomper MG. Quantification and minimization of mag- netic susceptibility artifacts on GRE images. J Comput Assist Tomogr 2000;24:958-64

8. Koch KM, Hargreaves BA, Pauly KB, et al. Magnetic resonance imaging near metal implants. J Magn Reson Imaging 2010;32:773-87

9. Kanal E, Barkovich AJ, Bell C, et al; Expert Panel on MR Safety. ACR guidance document on MR safe practices: 2013. J Magn Reson Imaging 2013;37:501-30

10. Jenkinson M, Beckmann CF, Behrens TE, et al. FSL. Neuroimage 2012;62:782-90

11. Filipek PA, Richelme C, Kennedy DN, et al. The young adult human brain: an MRI-based morphometric analysis. Cereb Cortex 1994;4: $344-60$

12. Shellock FG, Bedwinek A, Oliver-Allen M, et al. Assessment of MRI issues for a 3-T “immune" programmable CSF shunt valve. AJR Am J Roentgenol 2011;197:202-07

13. Shellock FG, Wilson SF, Mauge CP. Magnetically programmable shunt valve: MRI at 3-Tesla. Magn Reson Imaging 2007;25:1116-21

14. Vaccaro AR, Chesnut RM, Scuderi G, et al. Metallic spinal artifacts in magnetic resonance imaging. Spine (Phila $\mathrm{Pa}$ 1976) 1994;19: 1237-42

15. Khursheed F, Rohlffs F, Suzuki S, et al. Artifact quantification and tractography from 3T MRI after placement of aneurysm clips in subarachnoid hemorrhage patients. BMC Med Imaging 2011;11:19

16. Lavinio A, Harding S, Van Der Boogaard F, et al. Magnetic field interactions in adjustable hydrocephalus shunts. J Neurosurg Pediatr 2008;2:222-28

17. Toma AK, Tarnaris A, Grieve JP, et al. Adjustable shunt valve-induced magnetic resonance imaging artifact: a comparative study. J Neurosurg 2010;113:74-78

18. Kim MJ, Holodny AI, Hou BL, et al. The effect of prior surgery on blood oxygen level-dependent functional MR imaging in the preoperative assessment of brain tumors. AJNR Am J Neuroradiol 2005;26:1980-85

19. Peck KK, Bradbury M, Petrovich N, et al. Presurgical evaluation of language using functional magnetic resonance imaging in brain tumor patients with previous surgery. Neurosurgery 2009;64:644-52; discussion 652-53

20. Hargreaves BA, Worters PW, Pauly KB, et al. Metal-induced artifacts in MRI. AJR Am J Roentgenol 2011;197:547-55

21. Donahue MJ, Hoogduin H, van Zijl PC, et al. Blood oxygenation level-dependent (BOLD) total and extravascular signal changes and ÄR2* in human visual cortex at 1.5, 3.0 and 7.0 T. NMR Biomed 2011;24:25-34

22. Rane S, Mason E, Hussey E, et al. The effect of echo time and postprocessing procedure on blood oxygenation level-dependent (BOLD) functional connectivity analysis. Neuroimage 2014;95: $39-47$

23. Schmiedeskamp H, Newbould RD, Pisani LJ, et al. Improvements in parallel imaging accelerated functional MRI using multiecho echoplanar imaging. Magn Reson Med 2010;63:959-69

24. Shellock FG. Reference Manual for Magnetic Resonance Safety, Implants, and Devices: 2014 Edition. Los Angeles: Biomedical Research Publishing Group; 2014

25. Shellock FG. Metallic neurosurgical implants: evaluation of magnetic field interactions, heating, and artifacts at 1.5-Tesla. J Magn Reson Imaging 2001;14:295-99

26. Lövblad KO, Yilmaz H, Chouiter A, et al. Intracranial aneurysm stenting: follow-up with MR angiography. J Magn Reson Imaging 2006;24:418-22

27. Choi JW, Roh HG, Moon WJ, et al. Optimization of MR parameters of 3D TOF-MRA for various intracranial stents at 3.0T MRI. Neurointervention 2011;6:71-77

28. Donahue MJ, Strother MK, Hendrikse J. Novel MRI approaches for assessing cerebral hemodynamics in ischemic cerebrovascular disease. Stroke 2012;43:903-15 